\title{
Study on Multi-Level Layout Planning of Electric Vehicle Charging Stations Based on an Improved Genetic Algorithm
}

\author{
Maojing Jin ${ }^{\mathrm{a}}$, Ruifeng Shi ${ }^{\mathrm{b} *}$, Ning Zhang ${ }^{\mathrm{b}}$, Yang $\mathrm{Li}^{\mathrm{c}}$ \\ ${ }^{a}$ The High-Technology Research and Development Center, The Ministry of Science and Technology, Beijing 100044, China \\ ${ }^{b}$ School of Control and Computer Engineering, North China Electric Power University, Beijing 102206, China \\ ${ }^{c}$ Potevio New Energy co., Ltd. No.6 Haidian North Second Street,Haidian District,Beijing 100080, China
}

\begin{abstract}
Electric vehicle charging stations are essential infrastructure in developing electric vehicles. In order to construct reasonable amount of electric vehicle charging stations with proper locations, a mathematical multi-level layout planning model, which aims at minimizing the initial construction investment and the users' charging cost simultaneously, is employed in this paper. An improved genetic algorithm that takes an order based crossover operator, random mutation operator and meta-heuristic vehicles' allocation strategy is proposed to solve the planning problem. In order to evaluate the performance of the proposed algorithm, a case study is taken for verification and validation. Empirical results show that our proposed algorithm outperforms current result of the other algorithms, which demonstrates the effectiveness of the proposed algorithm on solving multi-level electric vehicles' charging station layout planning problems.
\end{abstract}

Keywords: Electric vehicle, charging station, multiple level planning, layout planning, genetic algorithms.

\section{Introduction}

Nowadays, a number of cities and areas begin construct electric vehicle (EV) charging stations in China to welcome the coming boom of EV industrialization. But the planning methods for the charging infrastructure layout has not well developed, which led to low efficiency and more financial investment. With the push on development of EV by the national government, charging stations construction become more and more important to support the dramatically increasing demands.

During the past decades, many efforts have been taken to improve the layout of EV charging station. Wang established a multi-objective planning model, which was based on the consideration of the characteristics of charging stations and/or charging users, charging demand distribution, power grid as well as the municipal planning [1]. Hatton studied the EV charging stations planning problem with consideration on current situation, ongoing developments, and future trends in the design and development of charging systems for battery electric vehicles, and specific requirements within the design of infrastructure systems for EVs were also taken into consideration [2]; Biao established the EV charging infrastructure construction evaluation model with the help of analytic hierarchy process for the functional domain and service window simultaneously [3]; Morrow analyzed the requirements of the charging infrastructure construction in three different regions, and an assessment model was also given to take the different charging infrastructure construction costs into consideration [4]; ReVelle reviewed the facilities layout optimization problems, in which discrete and continuous models were summarized [5]; Susan tried to solve facility layout problem by utilizing its stochastic/dynamic characteristics [6]; Zheng evaluated the merits and drawbacks of three different types of trade patterns, which combined the charging infrastructure construction and its operation into consideration [7].

\footnotetext{
* Manuscript received July 23, 2012; revised August 24, 2012.

Corresponding author. Tel.: +86-10-61772820; E-mail address: shi.ruifeng@126.com.
} 
In order to solve the general EV charging station layout problem, a multi-level EV charging station layout optimization model (MLEVCSL), which pursue to minimize the initial construction investment and users' charging cost simultaneously, is employed [8]. An improved genetic algorithm (IGA), which provides an order-based crossover and rule-based mutation operators, is proposed in this paper to solve the MLEVCSL problem. In order to verify the performance of the algorithm, an empirical case study is taken for demonstration.

\section{Multi-Level Electric Vehicle Charging Station Layout Planning Model}

The official guidance documents for constructing different levels of electric vehicle charging stations have been issued [9] (as shown in Table 1).

The charging stations levels and EV allocations can be affected by many reasons. The main factors should include initial construction investment; users' daily charging cost; vehicle retention; EV charging power; characteristics of power battery, etc.

Users' charging cost led by different charging behaviours, including the power consumption to arrive at the charging station and the loss due to occupied working time, is also taken into account in this paper. The multi-level EV charging station layout optimization model is based on two important aspects. One of the key factors is to calculate the users' cost, which is closely related to the distance from the EV's current place to the "ideal" charging station it is allocated. Another key factor is to get a well estimation on the initial construction investment. Thus the location selection and the allocation scheme for all EV candidates are two aspects to be dealt with properly to gain the optimal economic cost [8].

Table 1. Infrastructure construction standard for different levels of EV charging stations

\begin{tabular}{|c|c|c|c|c|}
\hline level of station & $\begin{array}{l}\text { battery storage } \\
\text { capacity/kWh }\end{array}$ & $\begin{array}{l}\text { distribution capacity of } \\
\text { single channel } / \mathrm{kVA}\end{array}$ & $\begin{array}{l}\text { the quant } \\
\text { big, medium-sized } \\
\text { commercial vehicle }\end{array}$ & $\begin{array}{l}\text { y service } \\
\text { passenger car }\end{array}$ \\
\hline Level 1 & $\geq 6800$ & $\geq 5000$ & $\geq 200$ & $\geq 500$ \\
\hline Level 2 & $3400 \sim 6800$ & $3000 \sim 5000$ & $100 \sim 200$ & $200 \sim 500$ \\
\hline Level 3 & $1700 \sim 3400$ & $1000 \sim 3000$ & $40 \sim 100$ & $100 \sim 200$ \\
\hline Level 4 & $\leq 1700$ & $\leq 1000$ & $\leq 40$ & $\leq 100$ \\
\hline
\end{tabular}

\subsection{Model assumptions}

- The candidate points of charging stations should take into account the demands' distribution and the environmental safety;

- The quantity of each demand point is proportional to the total number of the electric vehicles' charging demand in that area;

- In a fixed period of time, users in each demand point can only be allowed to charge their EVs at the station that it be allocated to;

- Users' charging cost is linearly positive correlation with the distance from the EV charging station, where Euclidean distance is employed in this paper.

\subsection{Mathematical model of a multi-level EV charging station layout planning problem}

$$
\begin{aligned}
& \min \quad C=\sum_{j} \sum_{m} F_{m} X_{j m}+\lambda \sum_{i} \sum_{j} D_{i} Z_{i j} d_{i j} \\
& \text { s.t. } \quad \sum_{j} Z_{i j}=1, \forall i \in I \\
& Z_{i j} \leq X_{j m}, \forall i \in I, j \in J, m \in M \\
& \sum_{i} D_{i} Z_{i j} \leq \sum_{m} C_{m} X_{j m}, \forall j \in J \\
& \sum_{m} X_{j m} \leq 1, \forall j \in J \\
& \sum_{j} \sum_{m} X_{j m}=P
\end{aligned}
$$




$$
\begin{aligned}
& X_{j m} \in\{0,1\}, \forall j \in J, m \in M \\
& Z_{i j} \in\{0,1\}, \forall i \in I, j \in J
\end{aligned}
$$

where $I$ is the demand points set, $i \in I$ refers to a demand point; $J$ is the candidate points set of charging stations, $j \in J$ refers to a candidate point; $D_{i}$ is the demand quantity at point $i$, namely the number of vehicles having charging demand; $F_{m}$ is the initial construction cost of station in level $m ; \lambda$ is the unit charging cost for user; $C_{m}$ is the service ability of station in level $m$, namely the number of serving vehicles daily; $d_{i j}$ is the distance from the demand point $i$ to the candidate point $j ; P$ is the quantity of station to be constructed; $X_{j m} \in\{0,1\}$, the value is 1 when the candidate point $j$ of station is constructed in level $m$, otherwise 0 ; and $Z_{i j} \in\{0,1\}$, the value is 1 when the users at demand point $i$ receive services at candidate point $j$, otherwise 0 .

In the above model, formula (1) gives the optimization objective, which aims to minimize the total planning cost, including the initial construction investment of charging stations and the users' charging cost in the later use. Formula (2) represents the users in each demand point have to arrive at an appointed charging station in a fixed period of time. Formula (3) represents the prerequisite conditions to make a candidate point into function. Formula (4) indicates that the charging demand quantity at one station must not exceed its maximum service ability. Formula (5) shows a candidate point must be constructed as only one of the levels. Formula (6) gives the constraint of total amount of charging stations to be constructed. Formula (7), (8) gives the feasible zones for the decision-making variables.

\section{Improved Genetic Algorithm for Multi-Level EV Charging Stations Layout Planning}

\subsection{Basic optimization principles of genetic algorithms}

Genetic algorithms search the optimal solutions with the basic rule of choosing the fittest one in the population evolution. During the process, outstanding individuals are given better fitness, which leads these individuals more choices to give offspring through crossover and mutation operators. Thus good features are preserved and bad ones are eliminated. Overall performance of the population continues to get improved, and eventually reach the optimal value [10]. When solving problems, genetic algorithms only take the advantage of the information of fitness function, but not any other conditions like continuation and differentiability. Thus it is particularly suitable for processing highly complex nonlinear problems.

\subsection{The improved genetic algorithm for multi-level electric vehicle charging stations layout planning}

In order to solve the problem described in formula (1)-(8), an improved genetic algorithm is proposed, which includes the main steps as the following:

Step 1. Population initialization. The initial population is randomly generated. According to the variable characteristics, binary coding strategy is employed in this study, which takes coding length as $J$. The number of 1 in the code is $P$ ( 1 means the point is selected to construct charging station), and the remaining ones can only be 0 to match the constraint.

Step 2. Calculate the fitness for each individual. When evaluating the performance of each scheme, additional work should be taken to make the solution a complete one: all the demand points should be allocated to the potential stations to finish the evaluation process.

Step 3: Bringing the next generation. In order to generate qualified offspring, specifically designed crossover and mutation operators are employed.

Step 4. Convergence. Either the best fitness of the generation doesn't improve during a continuous 50 generations or the maximum evolving generation is reached, the algorithm will output the best individual of that generation as the final result.

The flowchart of the improved genetic algorithm is shown in Fig. 1. 


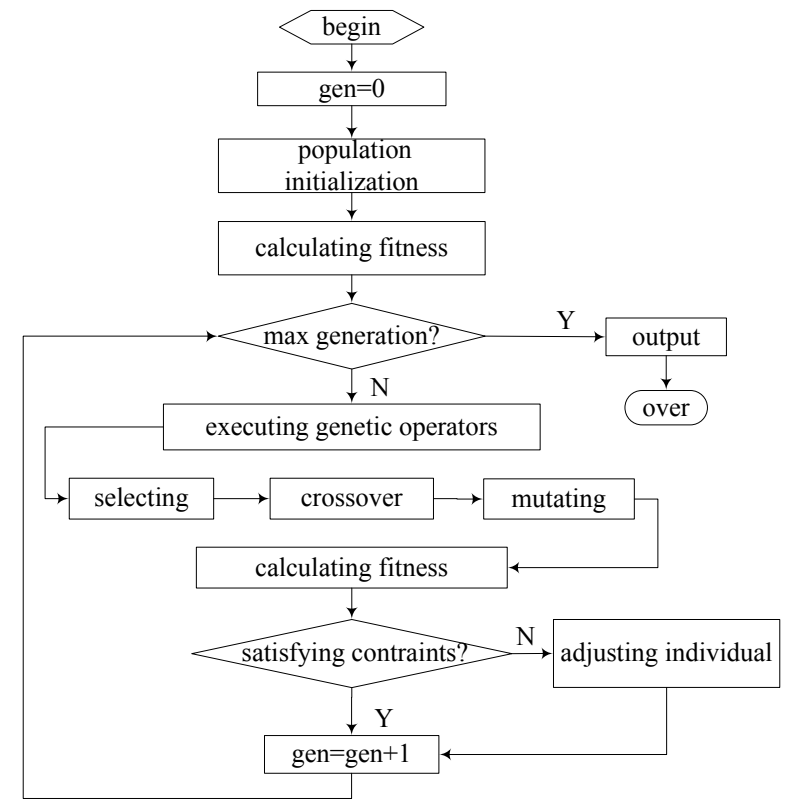

Fig. 1. Flowchart of the improved genetic algorithm

\section{Case Study}

\subsection{Problem description}

To verify and validate the performance of our proposed algorithm, an example, which is originally give in [8], is employed for case study. In a 50×50 zone, 30 charging demand users and 10 candidate points of charging stations are randomly generated. The aim of the layout planning is to choose 5 candidate points for constructing charging stations from the ten. The demand coordinates, the demand quantity and the candidate points coordinates are shown in Table 2 and Table 3 respectively. According to real application scenario, the users in each demand point are supposed to lay in the interval $(20,40)$. Besides, the 4 levels of charging stations are provided, and their service capability and initial construction investment are also estimated as shown in Table 4. The unit charging cost for one vehicle is assumed as a fixed value $\lambda=1 \mathrm{RMB} / \mathrm{km}=10^{-3} \times 1000 \mathrm{RMB} / \mathrm{km}$.

Table 2. Demand coordinates and their corresponding demands

\begin{tabular}{cccc|c|c|c|c}
\hline $\begin{array}{c}\text { Order } \\
\text { number }\end{array}$ & $X$ & $Y$ & Demands & $\begin{array}{c}\text { Order } \\
\text { number }\end{array}$ & $X$ & $Y$ & Demands \\
1 & 24.74 & 21.52 & 33 & 16 & 22.81 & 41.90 & 40 \\
2 & 45.68 & 8.01 & 35 & 17 & 27.11 & 4.62 & 33 \\
3 & 48.36 & 3.34 & 22 & 18 & 30.11 & 9.14 & 25 \\
4 & 6.34 & 5.79 & 29 & 19 & 27.44 & 7.81 & 28 \\
5 & 4.70 & 26.61 & 28 & 20 & 10.11 & 0.42 & 24 \\
6 & 20.87 & 41.78 & 24 & 21 & 20.05 & 18.98 & 30 \\
7 & 0.46 & 32.24 & 37 & 22 & 47.17 & 23.70 & 29 \\
8 & 10.79 & 45.46 & 37 & 23 & 41.00 & 2.87 & 30 \\
9 & 10.37 & 42.53 & 30 & 24 & 34.83 & 7.36 & 23 \\
10 & 4.02 & 40.93 & 40 & 25 & 3.17 & 36.14 & 22 \\
11 & 8.13 & 26.99 & 23 & 26 & 34.79 & 11.06 & 27 \\
12 & 21.86 & 27.01 & 38 & 27 & 25.66 & 28.79 & 33 \\
13 & 0.10 & 41.83 & 36 & 28 & 44.63 & 4.63 & 24 \\
14 & 0.99 & 4.92 & 29 & 29 & 41.09 & 49.70 & 33 \\
15 & 48.37 & 30.98 & 33 & 30 & 48.65 & 46.14 & 25 \\
\hline
\end{tabular}


Table 3.Charging stations candidate points' coordinates

\begin{tabular}{ccc}
\hline order number & $X$ & $Y$ \\
\hline 1 & 5.0 & 5.0 \\
2 & 6.0 & 32.0 \\
3 & 10 & 38 \\
4 & 25 & 25 \\
5 & 30 & 5 \\
6 & 43 & 8 \\
7 & 42 & 25 \\
8 & 45 & 40 \\
9 & 18 & 42 \\
10 & 32 & 15 \\
\hline
\end{tabular}

Table 4. Station levels and their corresponding construction investment

\begin{tabular}{ccc}
\hline Level & Service ability /(vehicles/day) & Sonstruction investment /(1000 RMB) \\
\hline 1 & 350 & 6500 \\
2 & 250 & 5300 \\
3 & 110 & 4000 \\
4 & 70 & 3500 \\
\hline
\end{tabular}

\subsection{Parameters setting and experiment scenario}

After experimental tests, the genetic algorithm's parameters are set as Table 5, and all the empirical studies are taken on a high performance PC with 4-core CPU $2.33 \mathrm{GHz}$, and $4 \mathrm{~GB}$ memory, with a python coded program.

\subsection{Results and discussion}

After the optimization process, we obtained the final solution as shown in Table 6, which is better than the result in literature [8] (shwon as Table 7). From the two solutions we can find that the layout solutions produced by the two algorithms have completely different proposals, either in the location of the station or in the choosing the station's level for the same candidate point. The result obtained in this study has saved almost 100, $000 \mathrm{RMB}$ for the whole project, which improves about $0.4 \%$ of the total cost.

Table 5. The parameters setting for the improved genetic algorithm

\begin{tabular}{ccccc}
\hline Parameters & Population size & Maximum generation & Probability of crossover & Probability of mutation \\
\hline Set value & 50 & 30 & 0.8 & 0.06 \\
\hline
\end{tabular}

Table 6. Results obtained with the IGA proposed in this paper

\begin{tabular}{cl}
\hline Optimal solution & \multicolumn{1}{c}{$\mathbf{1}, \mathbf{0 , 0 , 0 , 0 , 0 , 1 , 1 , 1 , 1 \}}$} \\
\hline & $1(3): 4,5,14,20 ;$ \\
& $7(4): 15,22 ;$ \\
Layout(level): distribution & $8(4): 29,30 ;$ \\
of demand points & $9(1): 6,7,8,9,10,11,12,13,16,25 ;$ \\
& $10(1): 1,2,3,17,18,19,21,23,24,26,27,28$ \\
\hline total cost (1000RMB) & 24010.099 \\
\hline
\end{tabular}

Table 7. Results from literature [8]

\begin{tabular}{cl}
\hline Optimal solution & \multicolumn{1}{c}{$\mathbf{1 0 , 1 , 0 , 0 , 1 , 0 , 1 , 1 , 1 , 0 \}}$} \\
\hline & $2(2): 4,5,7,10,11,13,14,25 ;$ \\
& $5(1): 1,2,3,17,18,19,20,21,23,24,26,28$ \\
Layout(level): distribution & $7(4): 15,22 ;$ \\
of demand points & $8(4): 29,30 ;$ \\
& $9(2): 6,8,9,12,16,27 ;$ \\
\hline total cost (1000RMB) & 24109.700 \\
\hline
\end{tabular}




\section{Conclusion}

Electric vehicle charging stations layout makes an irreplaceable role in the development of popularizing electric vehicles. An improved genetic algorithm, which takes advantage of the constraints of the locations choice and the users' allocation scheme, is proposed in this paper to solve a typical layout model. The result of our empirical case study has proved the effectiveness of our algorithm than the former one. Further attentions should be taken to consider the general multiple conflict objectives when making the layout decision.

\section{Acknowledgements}

The authors would like to thank the National Science Foundation of China (Grant Number 61203100), The Science Foundation of Hebei Province (Grant Number F2010001714) and the Fundamental Research Funds for the Central Universities (Grant Number 10MG27) for their financial supports with this research.

\section{References}

[1] Wang H, Huang Q, Zhang C, et al. A novel approach for the layout of electric vehicle charging station. In: Proc. of International Conference on Apperceiving Computing and Intelligence Analysi, 2010:64-67.

[2] Hatton CE, Beella SK, Brezet JC, et al. Charging station for urban settings: the design of a product platform for electric vehicle infrastructure in dutch cities. World Electric Vehicle Journal, 2009; (3):1-13.

[3] Li B, Chang X. Application of analytic hierarchy process in the planning of energy supply network for electric vehicles. Energy Procedia, 2011, 12:1083-1089.

[4] Morrow K, Karner D, Francfort J. Plug-in Hybrid Electric Vehicle Charging Infrastructure Review. Idaho Falls: Idaho National Laboratory, 2008.

[5] ReVelle CS, Eiselt HA. Location analysis: a synthesis and survey. European Journal of Operational Research, 2005, 165(1):1-19.

[6] Susan HO. Strategic facility Layout: a review. European Journal of Operational Research, 1998, 111:423-447.

[7] Zheng Z. Analysis on development trend of electric vehicle charging mode. In: Proc. of International Conference on Electronics and Optoelectronics (ICEOE 2011), 2011:440-442.

[8] Zhang G, Li B, Wang Y. The Layout and algorithm of multiple level for electric vehicle charging station. Journal of Shandong University, 2011;41(6):136-142. (in Chinese)

[9] Beijing administration of quality and technology supervision. In: DB11/Z 728-2010 Technical Specification of Electric Vehicle Power Supply and Security: Charging Station. Beijing: China Standards Press, 2010.

[10] Pelikan M, Goldberg DE. BOA: The Bayesian Optimization Algorithm. IlliGAL Report. 98013, Urbana, IL: Illionis Genetic Algorithms Lab, Univ Illinois, Urbana-Champaign, 1999. 